\title{
Structural damping with friction beams
}

\author{
L. Gaul, J. Roseira* and J. Becker \\ Institute of Applied and Experimental Mechanics, Universität Stuttgart, Pfaffenwaldring 9, 70550 Stuttgart, \\ Germany
}

Received 2007

Revised 2007

\begin{abstract}
In the last several years, there has been increasing interest in the use of friction joints for enhancing damping in structures. The joints themselves are responsible for the major part of the energy dissipation in assembled structures. The dissipated work in a joint depends on both the applied normal force and the excitation force. For the case of a constant amplitude excitation force, there is an optimal normal force which maximizes the damping. A 'passive' approach would be employed in this instance. In most cases however, the excitation force, as well as the interface parameters such as the friction coefficient, normal pressure distribution, etc., are not constant. In these cases, a 'semi-active' approach, which implements an active varying normal force, is necessary. For the 'passive' and 'semi-active' approaches, the normal force has to be measured. Interestingly, since the normal force in a friction joint influences the local stiffness, the natural frequencies of the assembled structure can be tuned by adjusting the normal force. Experiments and simulations are performed for a simple laboratory structure consisting of two superposed beams with friction in the interface. Numerical simulation of the friction interface requires non-linear models. The response of the double beam system is simulated using a numerical algorithm programmed in MATLAB which models pointto-point friction with the Masing friction model. Numerical predictions and measurements of the double beam free vibration response are compared. A practical application is then described, in which a friction beam is used to damp the vibrations of the work piece table on a milling machine. The increased damping of the table reduces vibration amplitudes, which in turn results in enhanced surface quality of the machined parts, reduction in machine tool wear, and potentially higher feed rates. Optimal positioning of the friction beams is based on knowledge of the mode shapes, which are obtained from experimental modal analysis. The modal damping and the natural frequencies for the two dominant modes are measured for several combinations of excitation force and normal force.
\end{abstract}

Keywords: Friction, joint, damping, vibration, semi-active, machine tool

\section{The double beam system}

The double beam is an academic example which demonstrates the main features of a friction damped system. It consists of two superposed beams (Fig. 1a) pressed together by one or more bolted joints. One end of the double beam is fixed. The friction between the beams is the dominant dynamical phenomenon. It influences the stiffness and the damping of the structure. Due to the simple nature of the test structure, measurements and simulations can be readily compared. The knowledge gained in the simple case of the double beam is then applied to the more complex machine tool case.

Figure $1 \mathrm{~b}$ shows an FE-model of both substructures. The corresponding mass and stiffness matrices were generated with the commercial FE-program ANSYS. The contact algorithm and the time-step integration were programmed as subroutines in MATLAB. The FE-model describes the normal and tangential contact of the double beam and allows the calculation of the friction forces $\boldsymbol{F}_{\mathrm{f}}$, which are added as external forces in the equation of motion,

*Corresponding author. Tel.: +49 71168154 ; Fax: +49 711 66282; E-mail: roseira@iam.uni-stuttgart.de. 
(a) Experimental setup

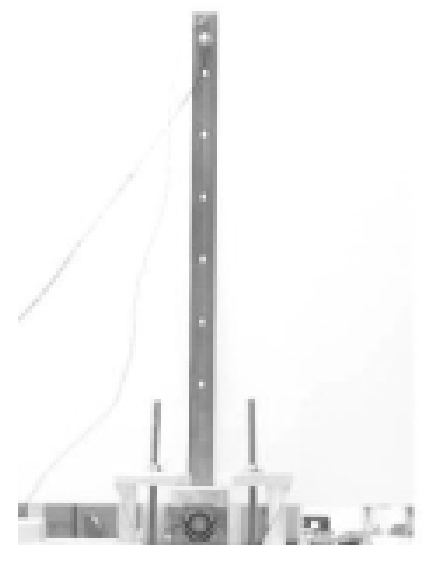

(b) FE discretization with external and contact forces

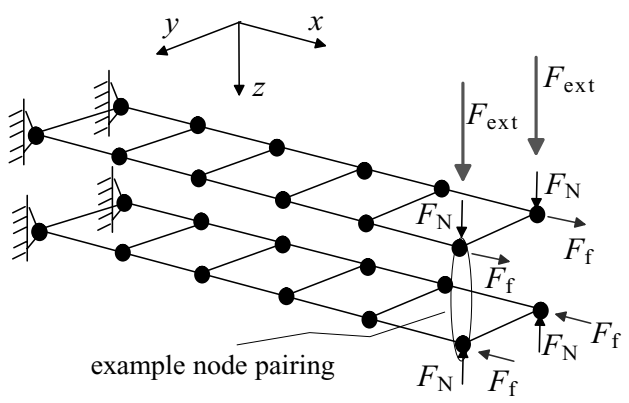

Fig. 1. Friction beam configuration.

$$
\left[\begin{array}{cc}
\boldsymbol{M}_{1} & \mathbf{o} \\
\mathbf{o} & \boldsymbol{M}_{2}
\end{array}\right]\left[\begin{array}{l}
\ddot{\boldsymbol{x}}_{1} \\
\ddot{\boldsymbol{x}}_{2}
\end{array}\right]+\left\{\left[\begin{array}{cc}
\boldsymbol{K}_{1} & \mathbf{o} \\
\mathbf{o} & \boldsymbol{K}_{2}
\end{array}\right]+\left[\begin{array}{ll}
\boldsymbol{K}_{11}^{\mathrm{N}} & \boldsymbol{K}_{12}^{\mathrm{N}} \\
\boldsymbol{K}_{21}^{\mathrm{N}} & \boldsymbol{K}_{22}^{\mathrm{N}}
\end{array}\right]\right\}\left[\begin{array}{l}
\boldsymbol{x}_{1} \\
\boldsymbol{x}_{2}
\end{array}\right]=\left[\begin{array}{l}
\boldsymbol{F}_{1}^{\text {ext }} \\
\boldsymbol{F}_{2}^{\text {ext }}
\end{array}\right]+\left[\begin{array}{l}
\boldsymbol{F}_{1}^{\mathrm{f}} \\
\boldsymbol{F}_{2}^{\mathrm{f}}
\end{array}\right] .
$$

In Eq. (1) $\boldsymbol{M}_{1}, \boldsymbol{M}_{2}$ are the mass matrices and $\boldsymbol{K}_{1}, \boldsymbol{K}_{2}$ are the stiffness matrices of the beams. $\boldsymbol{x}_{1}, \boldsymbol{x}_{2}$ are nodal displacements and rotations, and $\ddot{\boldsymbol{x}}_{1}, \ddot{\boldsymbol{x}}_{2}$ contain nodal accelerations and angular accelerations. $\boldsymbol{F}_{1}^{\text {ext }}, \boldsymbol{F}_{2}^{\text {ext }}$ are the external forces applied to beams 1 and 2, respectively. In the normal direction the superposed nodes are coupled with springs, which can have a linear or non-linear behavior, and are responsible for the no-separation and no-penetration conditions. The inclusion of a non-linear contact law was studied by [1,2]. This coupling is achieved with the matrices $\boldsymbol{K}_{11}^{\mathrm{N}}, \boldsymbol{K}_{12}^{\mathrm{N}}, \boldsymbol{K}_{21}^{\mathrm{N}}$ and $\boldsymbol{K}_{22}^{\mathrm{N}}$. In the tangential direction the coupling is stipulated by a friction model, which is used to calculate the friction forces $\boldsymbol{F}_{\mathrm{f}}$. The two beams were modeled using Bernoulli beam theory. The associated nodal displacements and rotations are illustrated in Fig. 2a. The relative displacement at the contact location is given by

$$
u=U_{2}-U_{1}=u_{2}+\frac{h}{2} \varphi_{2}-u_{1}+\frac{h}{2} \varphi_{1},
$$

and the relative velocity is given by

$$
v=\dot{u}_{2}+\frac{h}{2} \dot{\varphi}_{2}-\dot{u}_{1}+\frac{h}{2} \dot{\varphi}_{1}
$$

Hence, the contact problem is described based on the motion of the neutral beam axes. Under the assumption of small displacements it follows that the node pairing between the two beams does not need to be rearranged during the course of the simulation. Thus, there is no need for a contact detection algorithm which strongly reduces the calculation effort. A representative node pairing is depicted in Fig. 1b.

There are several friction models available for calculation of the tangential contact: static models, such as the Coulomb friction model for example, or dynamic models, such as the Dahl and LuGre friction models [3]. It is possible to program several contact models as subroutines. The numerical simulations where carried out with the Masing friction model. This model generalizes the Coulomb friction with finite contact stiffness $k_{\mathrm{T}}$ (Fig. 2b). This model allows a better representation of the measured hysteresis. The friction force is governed by the Masing evolution rule

$$
\dot{F}_{\mathrm{f}}=k_{\mathrm{T}} v \frac{1}{2}\left\{1-\operatorname{sgn}\left(F_{\mathrm{f}}^{2}-F_{\mathrm{C}}^{2}\right)-\operatorname{sgn}\left(v F_{\mathrm{f}}\right)\left[1+\operatorname{sgn}\left(F_{\mathrm{f}}^{2}-F_{\mathrm{C}}^{2}\right)\right]\right\} .
$$

Experiments on a resonator with a friction joint allow the determination of the contact stiffness as a function of the normal force [4], via a measured hysteresis characteristic. The slope of the hysteresis in the sticking phase is the sought contact stiffness. The regularized form of the Masing Evolution Rule 
(a) Node-to-node contact model

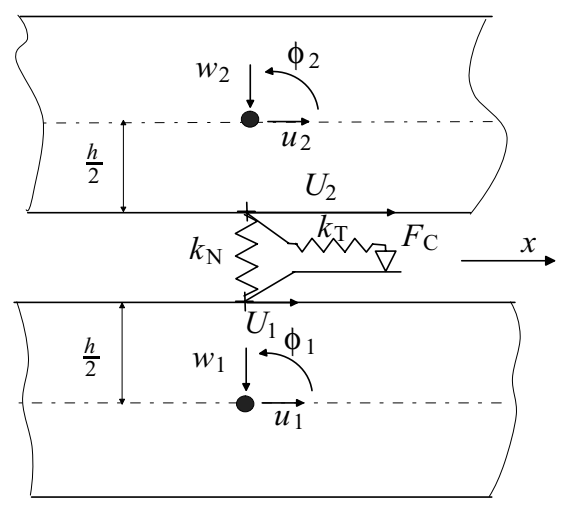

(b) Hysteresis of the Coulomb and Masing Models

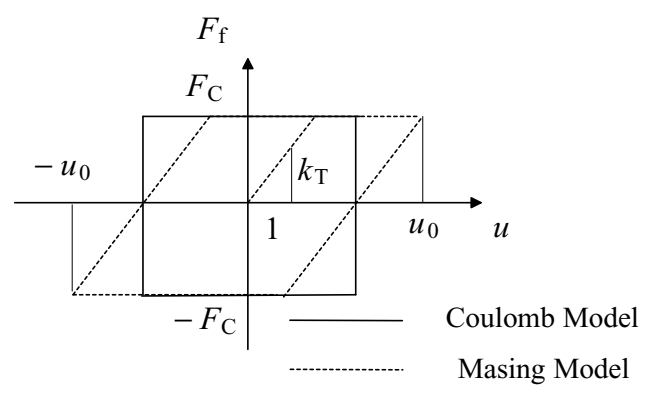

Fig. 2. Contact model.

$$
\dot{F}_{\mathrm{f}}=k_{\mathrm{T}} v\left\{1-\frac{1}{2}\left(\frac{F_{\mathrm{f}}}{F_{\mathrm{C}}}\right)^{m}\left[1+\operatorname{sgn}\left(v F_{\mathrm{f}}\right)\right]\right\}
$$

more closely approximates the measured hysteresis characteristic, where $m$ is a smoothing parameter. The optimal value of the smoothing parameter, $m=8$, was determined from the resonator mass experiment in [4]. This experimentally determined parameter is used in the numerical simulations of the double beam. A Masing friction model with multiple springs for the modeling of joints was formulated in [6].

The node-to-node model can also be used for 2D contact problems. For that purpose the displacements and rotations are considered in the $x$ - and $y$-directions. The tangential contact forces are also divided in these two directions. This formulation is applied to the case of plate elements with nodes in the middle layer. By that the 1D contact problem is generalized for 2D. Another possible formulation for the 2D contact problem can be found in [1, 8], where there are contact algorithms using zero thickness elements.

In Fig. 3a, the displacement in $z$-direction of the free end of the double beam in Fig. 1 a is plotted as a function of time. The free vibration is divided in two phases: the sliding and the sticking phase. In the sliding phase there is energy dissipation, since the relative displacement $u$ in the contact layer generates dissipative friction forces, which cause a reduction of the vibration amplitude. The energy dissipated per cycle corresponds to the enclosed area of the hysteresis characteristic in Fig. 3. The friction forces $F_{\mathrm{f}}$ not only depend on the magnitude of the relative displacements, but also on the applied normal force $F_{\mathrm{N}}$. Because of the weak coupling of the double beam in this phase, the stiffness and eigenfrequency coincide with those of a single beam. For high normal forces both beams stick, which results in increased stiffness and natural frequencies. Because the relative displacements are zero, no energy is dissipated. In this phase the vibration amplitude remains constant.

For the same ramp load ( $\max .20 \mathrm{~N}$ ) applied at the free end with subsequent release, the double beam has a lower initial deflection (Fig. 3) for high normal force $(8 \mathrm{kN})$ than for low normal force $(1 \mathrm{kN})$. This is due to the higher stiffness caused by tangential sticking. For high normal forces, the double beam behaves like a single beam whose thickness is twice that of the constituent beams.

Near the fixed end of the double beam there is mainly sticking. Near the free end large relative displacements occur. Therefore, if several friction joints are available and in order to maximize the energy dissipation, the normal force should be higher near the free end than near the fixed end.

The maximum energy dissipation takes place when the normal force reaches the limit just before sticking occurs. This is the main task of a control algorithm [7,8]. Another function of this algorithm is to achieve a fast and economic adjustment of the normal force whenever the contact characteristics (like coefficient of friction, corrosion, etc.) are altered.

For model validation, experimental and numerical results were compared. The measured and computed relative velocities were compared in the time and frequency domain. The experimental velocity was obtained via integration of the acceleration signal. The measurement of the normal forces was carried out with ring load cells placed in the bolted joints. The signal processing was achieved using a PC-based FFT analyzer. 
(a) Displacement

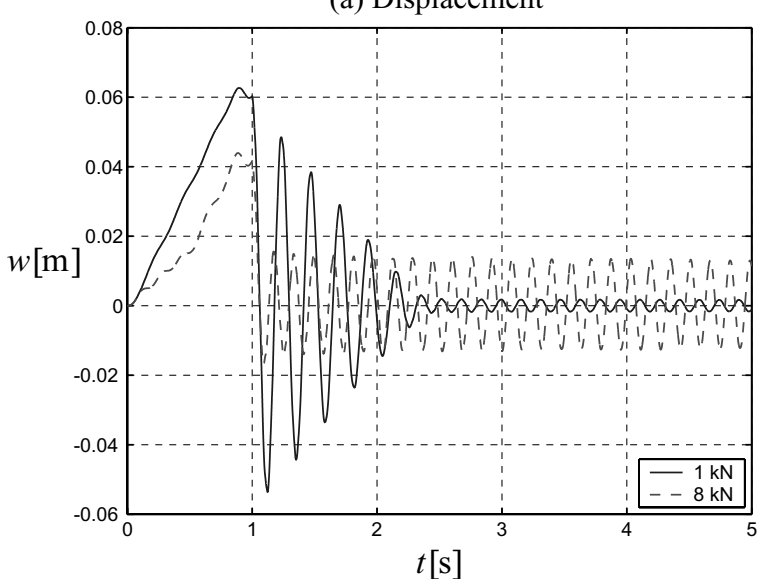

(b) Hysteresis

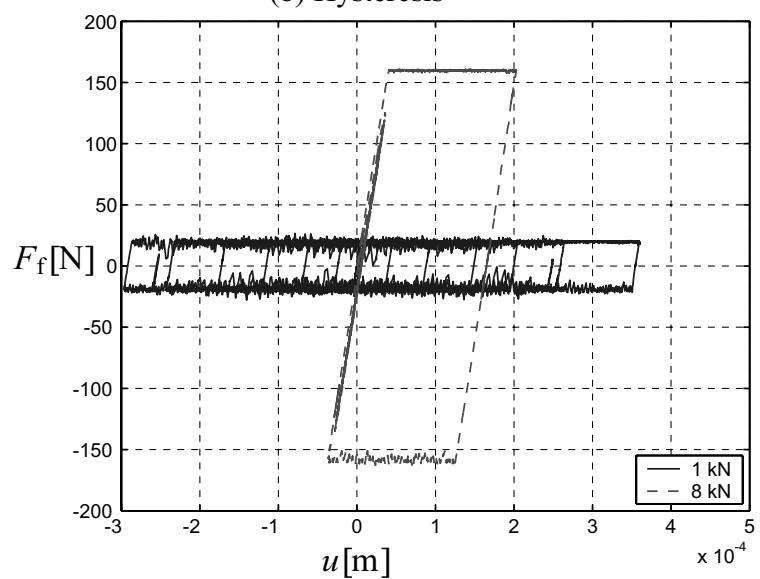

Fig. 3. Comparison of the free vibration and hysteresis for two normal forces (both numerical simulations).
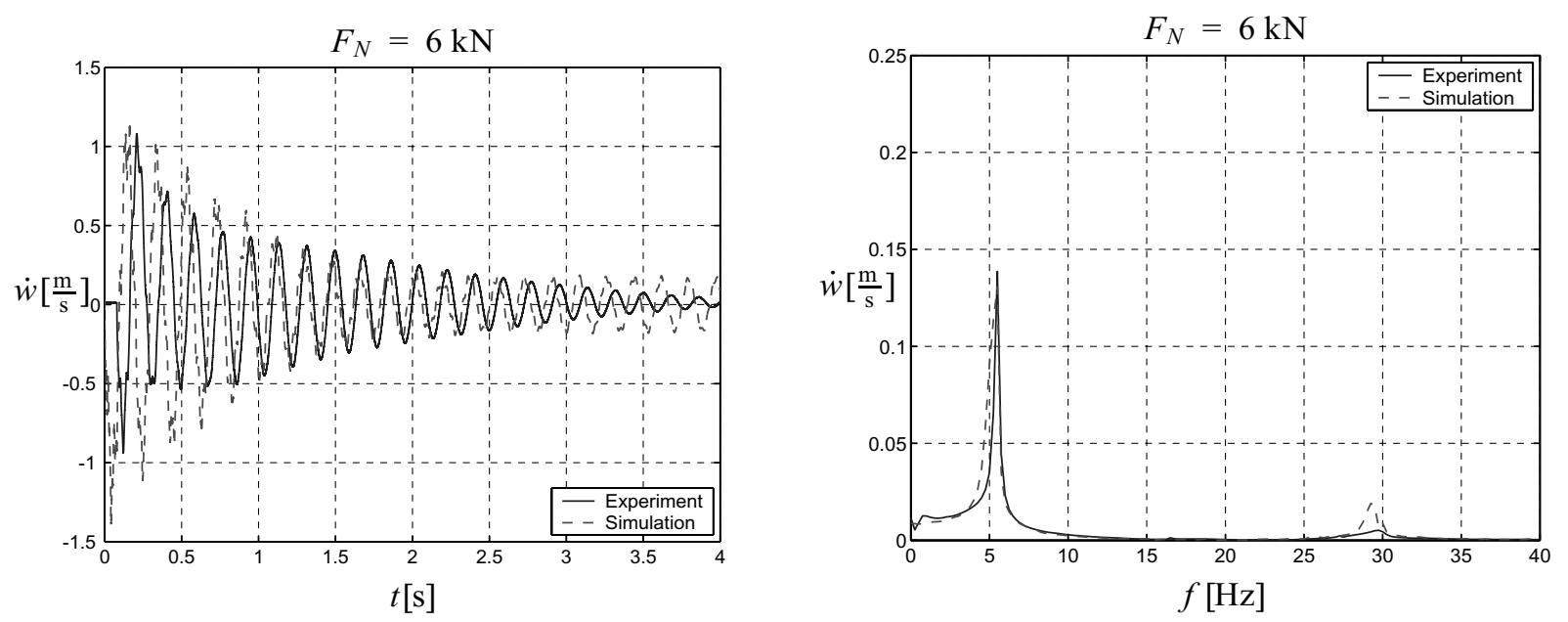

Fig. 4. Time- and frequency-domain comparison between numerical and experimental velocities for the case of a freely-vibrating double beam with contact normal force $F_{N}=6 \mathrm{kN}$.

At the free end of the double beam, a weight of $20 \mathrm{~N}$ was applied and then set free (snap-back excitation). Figure 4 shows a comparison of simulated and measured velocities of the free vibration. There is a good agreement between simulation and experiment in both time and frequency domain. (Note: in the measured velocity curve a certain exponential decay can be observed. This is due to damping mechanisms other than friction, that were not taken into consideration in the numerical model, for example material damping).

\section{Experimental validation of the friction beam concept in a machine tool}

The objective of an application of the friction beam in the machine tool HERMLE UWF $900 \mathrm{~W}$ (Fig. 5a) is to damp undesired vibrations which can compromise the machining performance. With the concept of optimal placed bolted friction beams there will be an improvement of the damping characteristics, and through stiffness tuning, resonance problems will be avoided.

In order to identify the practicability of the concept, a friction beam was attached to the work piece table of the milling machine (Fig. 5b). With experimental modal analysis (EMA) the vibration modes were identified in 
(a) Analyzed milling machine HERMLE UWF $900 \mathrm{~W}$

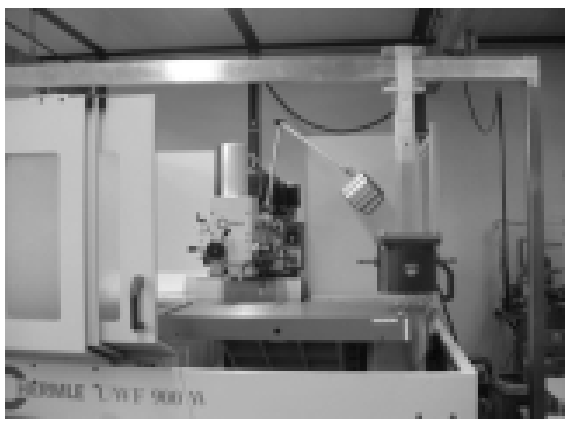

(b) Front view (above) and top view (below) of the work piece table
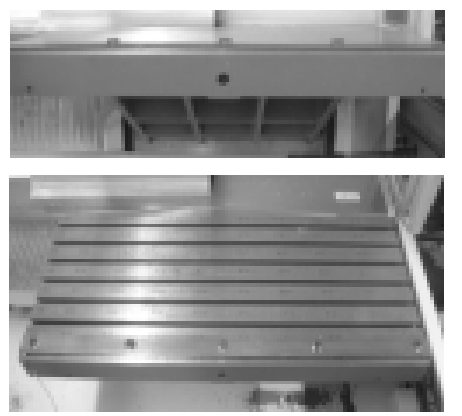

Fig. 5. Analyzed milling machine and work piece table with attached friction beam.

(a) Mode 1 at $500 \mathrm{~Hz}$

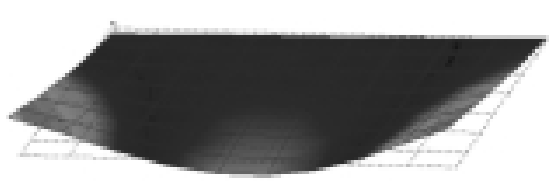

(b) Mode 3 at $1000 \mathrm{~Hz}$

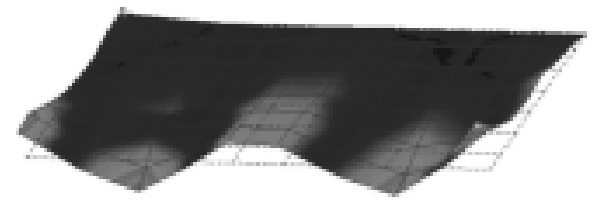

Fig. 6. Mode 1 (a) and 3 (b) of the work piece table of the milling machine.

order to determine the effect of the friction beam on the natural frequencies and modal damping ratios. (Under the assumption of weak nonlinear behavior, EMA can be used for determining the linearized dynamic characteristics of a structure).

Since the work piece table is made of gray cast iron and due to the rib stiffeners, it has inherently high modal damping, making a further enhancement in damping very difficult. In softer substructures the damping improvement is easier.

\subsection{Experimental modal analysis of the work piece table with impulse hammer}

The first set of measurements was made using an impulse hammer as excitation. A 4 channel PC measurement board acquisition system with a FFT Analyser was used for data collection. The response of the table is measured by accelerometers. The normal force in the bolted joints is measured with ring load cells. The EMA was carried out using 80 hammer points and 2 accelerometers. A schematic of the work piece table with attached friction beam is depicted in Fig. 7a. The normal force between work piece table and friction beam is adjusted at two variable friction joint locations.

An EMA was first carried out without the friction beam to determine the natural frequencies, mode shapes and damping of the original structure. Figure 6 shows two dominant modes of the work piece table.

The friction beam is placed in the area where the largest bending displacements occur, which is also where the largest relative displacements take place. Here, the maximal enhancement of damping can be achieved. Table 1 shows the shift of the eigenfrequencies as well as the increment in the modal damping due to the stiffness, damping and inertial effects of the friction beam for several normal forces $F_{\mathrm{N}}$. The modal damping for mode 1 is tripled when a low normal force of $F_{\mathrm{N}}=1 \mathrm{kN}$ is applied to the friction beam, thus proving the efficiency of the concept. A controlled concept is expected to have an even more pronounced effect $[3,5,7,8]$.

\subsection{EMA of the work piece table with shaker}

A new set of measurements was carried out using shaker excitation (Fig. 5a). The objective of this alternative experimental setup is to define the relation between the excitation force level and the optimal normal force. With 
Table 1

Eigenfrequencies and modal damping of the work piece table with and without friction beam for several normal forces

\begin{tabular}{cccc}
\hline Friction beam & $F_{\mathrm{N}}$ & 1. Eigenfrequency & Modal damping \\
\hline no & - & $499 \mathrm{~Hz}$ & $0.638 \%$ \\
yes & $10 \mathrm{kN}$ & $509 \mathrm{~Hz}$ & $0.738 \%$ \\
yes & $5 \mathrm{kN}$ & $507 \mathrm{~Hz}$ & $0.726 \%$ \\
yes & $1 \mathrm{kN}$ & $492 \mathrm{~Hz}$ & $1.8 \%$ \\
\hline
\end{tabular}

(a) for modal analysis with impulse hammer

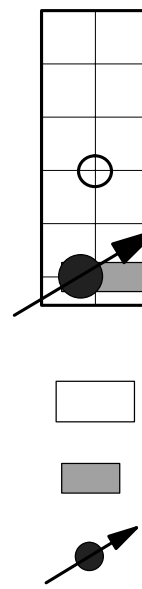

(b) for modal analysis with shaker

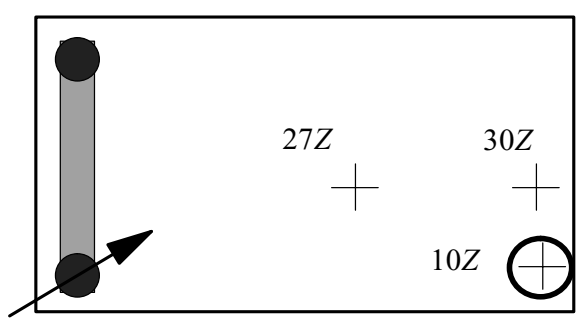

fixed friction joint

excitation point

measuring point

Fig. 7. Studied configurations of the friction beams.

an impulse hammer it is not possible to obtain an arbitrary level of excitation force, since it depends on the impact made by a human. With shaker excitation it is possible to define a desired excitation level, which is to be applied in the structure.

An alternative configuration of the friction beam is studied: the friction beam is applied on one side of the work piece table as illustrated in Fig. 7b. It should be noted that excitation and response points are located far from the friction beam. The friction beam affects not only the local but also the global stiffness and damping. The two dominant modes, the first and the third, were studied.

The excitation signal from the FFT analyzer is amplified and is then used to drive the electrodynamic shaker. The shaker is attached to the work piece table using a movable support frame as shown in Fig. 5a. The excitation force is measured directly at the shaker tip with a load cell.

For mode 1 (Fig. 6a), a sweep signal from $200 \mathrm{~Hz}$ to $850 \mathrm{~Hz}$ and with amplitudes varying from $6 \mathrm{~N}$ to $40 \mathrm{~N}$ was used to excite the structure around the resonance frequency of $500 \mathrm{~Hz}$. Normal forces from $25 \mathrm{~N}$ to $1200 \mathrm{~N}$ were applied in the variable friction joint. Figure 8 a depicts the modal damping of mode 1 as a function of the excitation force amplitude $F_{E}$ and normal force $F_{N}$. For very low normal forces (less than $25 \mathrm{~N}$ ), the modal damping remains low, because the friction beam and the working table are practically uncoupled. For intermediate normal forces (from 25-200 N) and for an excitation level of $30 \mathrm{~N}$, the highest modal damping is achieved. For $F_{N}=100 \mathrm{~N}$ it is possible to see the influence of the excitation level on the achieved modal damping. The modal damping increases until $F_{E}=30 \mathrm{~N}$ and starts to decrease after that. This change is due to the onset of the macro slip phase. On the contrary, for a normal force of greater than $200 \mathrm{~N}$, the system enters the sticking phase and, therefore, the modal damping decreases. It is expected that for higher excitation levels (greater than $40 \mathrm{~N}$ ), which the used shaker is not capable of achieving, the optimal normal force also increases. We can therefore conclude, that for high excitation levels, high normal forces should be applied. Figure $8 \mathrm{~b}$ illustrates the influence of normal force and excitation force on the first natural frequency. The eigenfrequency drops when the modal damping rises. For high normal forces the eigenfrequency tends to stabilize when the system enters the sticking phase. This stabilization can happen below or above the original eigenfrequency, depending on whether the friction joint has a predominant mass or stiffness 
(a) Modal damping as a function of $F_{E}$ and $F_{N}$ - mode 1

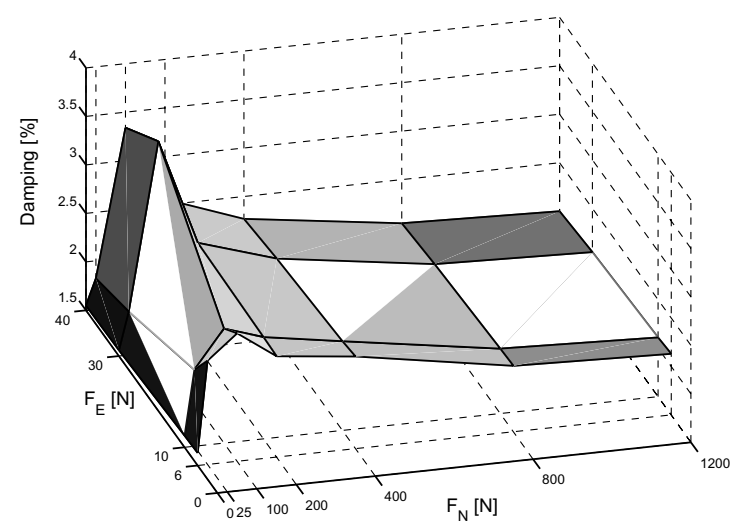

(b) Eigenfrequency as a function of $F_{E}$ and $F_{N}$ - mode 1

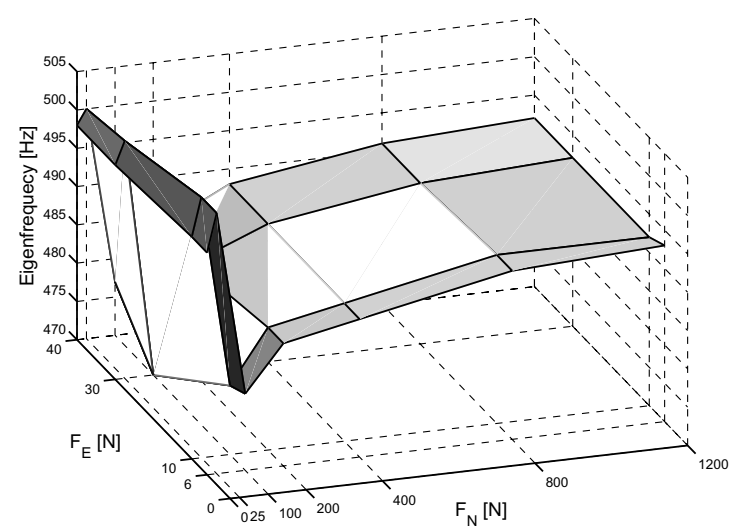

Fig. 8. Modal damping and eigenfrequency for the work piece table with friction beam at one side - mode 1 .

(a) Modal damping as a function of $F_{E}$ and $F_{N}$ - mode 3

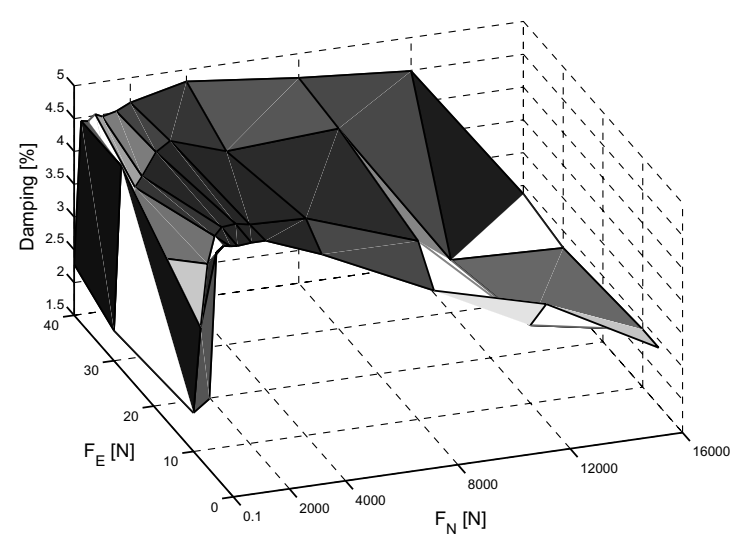

(b) Eigenfrequency as a function of $F_{E}$ and $F_{N}$ - mode 3

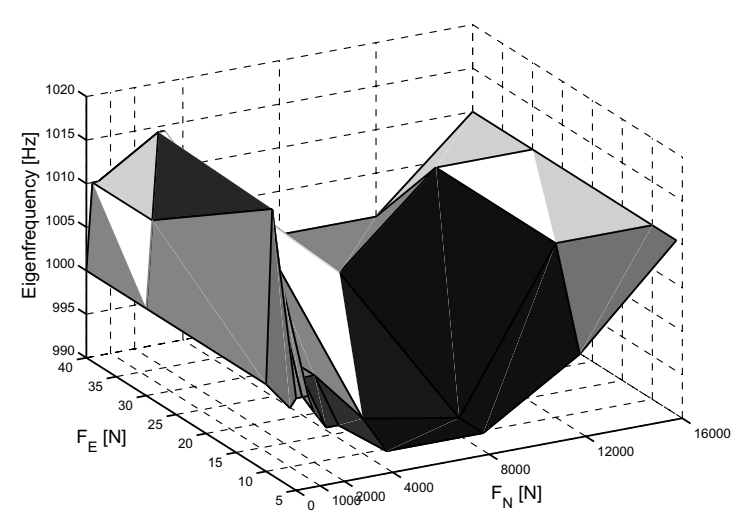

Fig. 9. Modal damping and eigenfrequency for the work piece table with friction beam at one side - mode 3 .

effect, respectively. In this case the eigenfrequency of the working table with friction joint in the sticking phase is below $500 \mathrm{~Hz}$, namely $495 \mathrm{~Hz}$. It is very difficult to predict the frequency shift. Certain is only that the frequency shift due to the friction joint exists. The direction and size of the frequency shift depends on several factors, such as geometry, area of application, analyzed mode and boundary conditions. For mode 3 at $1000 \mathrm{~Hz}$ (Fig. 6b) a sweep signal between 700 and $1350 \mathrm{~Hz}$ was applied to the structure. The excitation signal amplitudes varied from $6 \mathrm{~N}$ until $40 \mathrm{~N}$ just as in the mode 1 case. Figure 9 a shows the modal damping of mode 3 as a function of the excitation force amplitude $F_{E}$ and normal force $F_{N}$. In this case, the normal forces were varied between 0 and $12000 \mathrm{~N}$, since the interesting damping interval is located at higher normal forces. As in the mode 1 case, the eigenfrequency decreases in the high modal damping interval. As opposed to the mode 1 findings, the eigenfrequency tends to increase when the system enters the sticking phase for high normal forces, which means that the stiffness effect is dominant.

\section{Conclusions}

Friction beams can be used not only as efficient structural dampers, but also as eigenfrequency tuners. They can be placed easily in a vast field of structures, not only in the project development phase, but also in those already in operation. For proper application, careful dimensioning of the friction beams should be carried out. Stiffness and 
mass, as well as positioning and operating normal forces should be defined as a function of the excitation. This task is facilitated with appropriate numerical simulation, although the final validation should be carried out with measurements, in particular with EMA. The application of friction beams leads to reduced vibration amplitudes, which ultimately translates into enhanced surface quality, reduced sound radiation, and longer machine tool life. The simplicity, flexibility, efficiency, and low cost of friction beams makes them a highly competitive means for enhancing structural damping.

\section{Acknowledgements}

The financial support of the project 'Adaptive Friction Surfaces for Vibration Damping in Machine Tools' by the Deutsche Forschungsgemeinschaft DFG in the framework of the Priority Program 1156 'Adaptronics in Machine Tools' is gratefully acknowledged.

\section{References}

[1] L. Gaul and M. Mayer, Modelling of Contact Interfaces using Segment-to-Segment-Elements for FE Vibration Analysis, Proc. Intl. Modal Analysis Conference IMACXXIII, Session: Modelling of Structure Joints, Organizer L. Gaul, Orlando, 2005.

[2] M. Mayer and L. Gaul, Segment-to-segment contact elements for modelling joint interfaces in finite element analysis, Mechanical Systems and Signal Processing 21 (2007), S724-S734.

[3] L. Gaul and R. Nitsche, Friction Control for Vibration Suppression, Mechanical Systems and Signal Processing 14(2) (2000), S139-S150.

[4] L. Gaul and J. Lenz, Nonlinear dynamics of structures assembled by bolted joints, Acta Mechanica 125(1-4) (1997), S169-S181.

[5] L. Gaul, H. Albrecht and J. Wirnitzer, Semi-active friction damping of large space truss structures, Schock and Vibration 11 (2004), S. $173-186$

[6] VDI-Richtlinie 3830 Blatt 3 Kap. 2: “Dämpfung an Fügestellen.” VDI-Handbuch Schwingungstechnik, 2004.

[7] J. Becker and L. Gaul, Semi-active damping of vibrations of mechanical structures with friction dampers, Proceedings of the 13th International Conference of Sound and Vibration, 2006.

[8] L. Gaul, K. Willner, J. Roseira and J. Becker, Adaptive Reibflächen zur Schwingungsreduktion an Werkzeugmaschinen, Antrag im Rahmen des Deutsche Forschungsgemeinschaft Schwerpunktprogrammes 1156-Adaptronik fir Werkzeugmaschinen, 2005. 

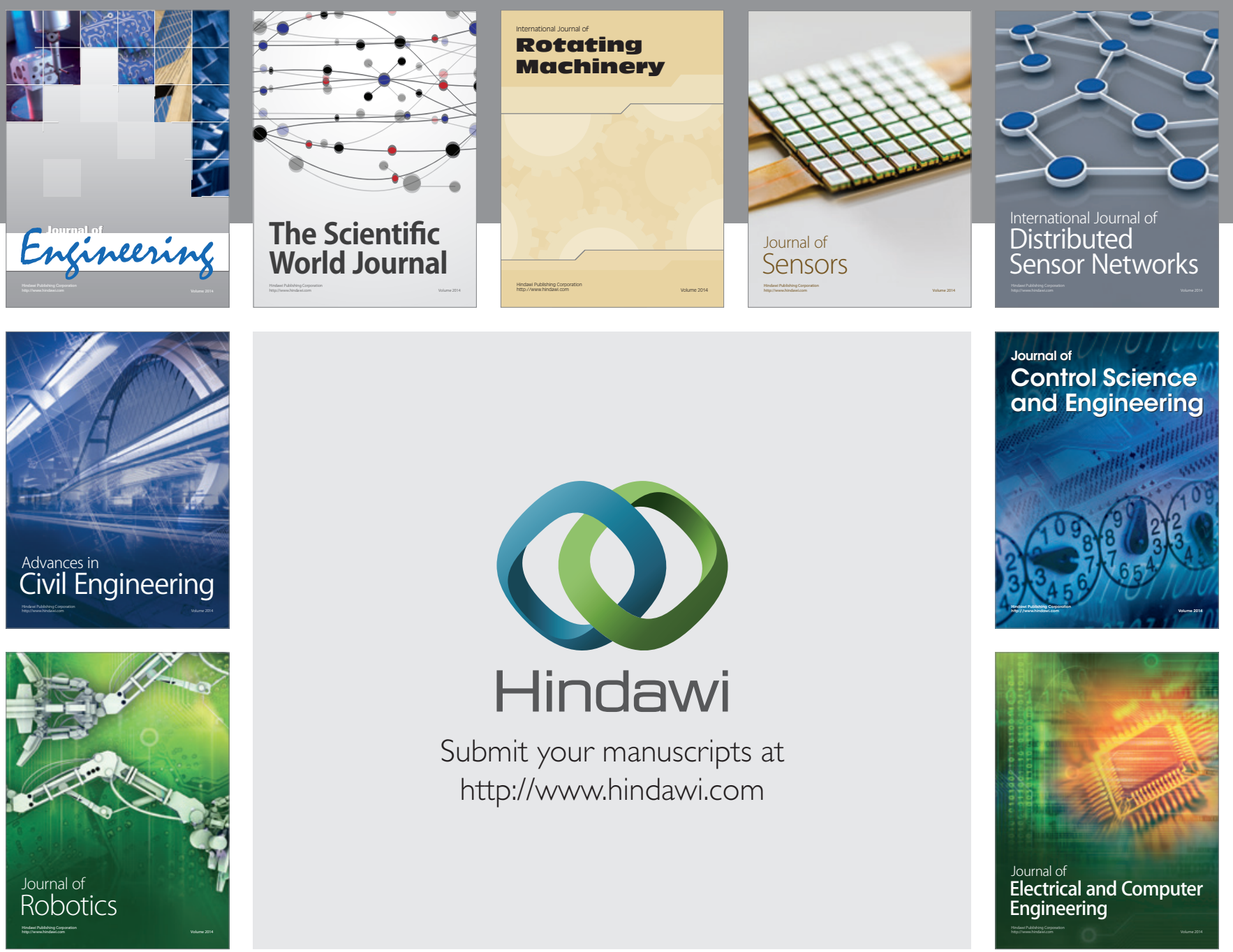

Submit your manuscripts at

http://www.hindawi.com
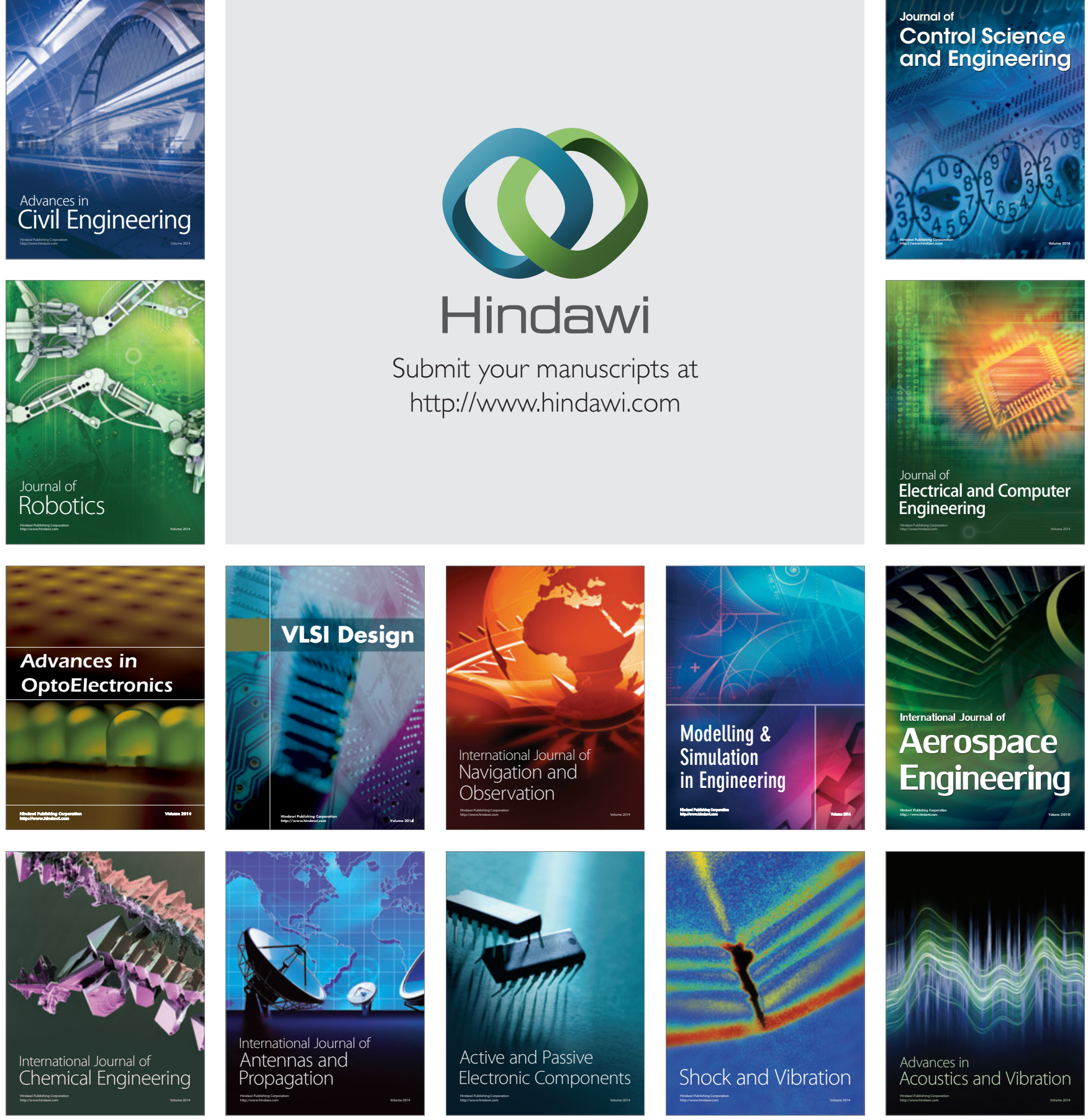